\title{
The preventive effects of ascorbic acid supplementation on locomotor and acetylcholinesterase activity in an animal model of schizophrenia induced by ketamine
}

\author{
LOUYSE S. DAMAZIO, FLÁVIA R. SILVEIRA, LARA CANEVER, ADALBERTO A. DE \\ CASTRO, JADNE M. ESTRELA, JOSIANE BUDNI and ALEXANDRA I. ZUGNO
}

Programa de Pós-Graduação em Ciências da Saúde, Laboratório de Neurociências, Universidade do Extremo Sul Catarinense, Unidade Acadêmica de Ciências da Saúde, Av. Universitária, 1105, Bairro Universitário, 88806-000 Criciúma, SC, Brazil

Manuscript received on August 1, 2016; accepted for publication on October 26, 2016

\begin{abstract}
Studies have shown that schizophrenic patients seem to have nutritional deficiencies. Ascorbic acid (AA) has an important antioxidant effect and neuromodulatory properties. The aim of this study was to evaluate the effects of AA on locomotor activity and the acetylcholinesterase activity (AChE) in an animal model of schizophrenia (SZ). Rats were supplemented with AA $(0.1,1$, or $10 \mathrm{mg} / \mathrm{kg}$ ), or water for 14 days (gavage). Between the 9th and 15th days, the animals received Ketamine (Ket) $(25 \mathrm{mg} / \mathrm{kg}$ ) or saline (i.p). After the last administration (30 $\mathrm{min}$ ) rats were subjected to the behavioral test. Brain structures were dissected for biochemical analysis. There was a significant increase in the locomotor activity in Ket treated. AA prevented the hyperlocomotion induced by ket. Ket also showed an increase of AChE activity within the prefrontal cortex and striatum prevented by AA. Our data indicates an effect for AA in preventing alterations induced by Ket in an animal model of SZ, suggesting that it may be an adjuvant approach for the development of new therapeutic strategies within this psychiatric disorder.
\end{abstract}

Key words: Schizophrenia, ascorbic acid, ketamine, hyperlocomotion, Acetylcholinesterase.

\section{INTRODUCTION}

Schizophrenia (SZ) is a chronic and debilitating neuropsychiatric disorder, considered to be a major cause of chronic disability which affects about $1 \%$ of the worldwide population (Javitt 2010, Boison et al. 2012). The World Health Organization (WHO) reports seven new cases per year for every 10.000 inhabitants, with the incidence in males (5.7 cases) being higher than in females (4.6 cases) (Mari and Leitão 2000). It usually occurs in the late teenage

Correspondence to: Alexandra Ioppi Zugno

E-mail: alz@unesc.net years or in early adulthood (Marsman et al. 2013), and even with few concrete results relating to the etiology and the underlying mechanisms of the disorder, the current literature indicates that its development is related to prenatal exposure and / or perinatally to various environmental insults. These include maternal exposure to stress, infection and/ or immune activation, nutritional deficiencies and other obstetric complications (Baguelin-Pinaud et al. 2010, Meyer and Feldon 2010), the presence of genes which can increase the susceptibility to the disorder in conjunction with environmental factors 
and changes in brain development (Ayhan et al. 2010, Nagai et al. 2011).

The symptoms of SZ are divided into three categories: positive symptoms (e.g. occurrences of psychosis, delirium, hallucinations and disorganized thoughts); negative symptoms (e.g. social and emotional isolation, affective blunting and anhedonia) and cognitive deficits (e.g. working memory, disorganization, disorientation and lack of attention) (Bowie and Harvey 2006). Schizophrenic patients are often found to have cognitive dysfunctions which are related to changes in the cholinergic system. Acetylcholine (ACh) is a cholinergic neurotransmitter that is synthesized in the presynaptic neuron by choline acetyltransferase from the choline and acetyl-coenzyme $\mathrm{A}$, and this neurotransmitter has a fundamental role in motor function, cognitive function and memory (Voss et al. 2008). Acetylcholinesterase (AChE) is an enzyme that is important in controlling the transmission of nerve impulses through the cholinergic synapse, since it is responsible for the hydrolysis and inactivation of ACh to choline and acetate (Soreq and Seidman 2001).

It has been observed that the prefrontal cortex, hippocampus and striatum of schizophrenic patients show reductions in the density of nicotinic alpha-7 Ach receptors (Freedman 2003), and changes in the levels of Ach, which are both associated with the cognitive dysfunctions seen in SZ (Meltzer and McGurk 1999). This evidence indicates that schizophrenic patients present with an imbalance in the cholinergic system, such as reductions of ACh in their brain structures (Bencherif et al. 2012).

Basic research in animals is a promising tool being used to gain a deeper understanding of this disorder, allowing researchers to evaluate and establish new strategies for its prevention, and helping to develop new pharmacologic therapies adjunct to the present treatments (Meyer and Feldon 2010). Standing out amongst the pharmacological animal models of SZ are those which use the administration of non-competitive antagonists of glutamate receptors N-methyl d-aspartate (NMDA), such as ketamine (Ket) (Canever et al. 2010, De Oliveira et al. 2011). These are responsible for pore blockage within the NMDA channel, which prevents the influx of calcium and can induce a transient and reversible psychotic state, including the positive, negative and cognitive symptoms of SZ in healthy subjects (Mecheri et al. 2001). In rodents, these drugs also induce the characteristic behaviors seen in SZ, such as hyperactivity, stereotypy, changed social behavior and sensory or cognitive deficits (Lipska and Weinberger 2000).

As well as the environmental and genetic factors, we should also take into account the act of feeding. Over the years, research has been conducted focusing on the nutritional status of schizophrenic patients, with high-impact studies paying particular attention to possible deficiencies in some vitamins. It is understood that patients with chronic SZ also have low levels of ascorbic acid (AA) (Milner 1963, Schorah et al. 1983). AA is a water soluble vitamin that is found in high concentrations within the central nervous system (CNS). It presents an antioxidant action and acts as an essential reducing agent for the activity of various enzymes. It is also involved in neuronal regulation, providing an essential action within the brain, while also playing a major role in neuronal regulation (Smythies 1996, 2000). The shortage of this vitamin in schizophrenic patients occurs as a metabolic response to the inflammatory aspects of the disease, increasing the clearance of AA and reducing plasma concentrations to undetectable levels in some cases (Rumelin et al. 2005).

Besides the pathological processes within the disorder which increase the metabolism of the vitamin (Reddy et al. 2003), these patients require careful attention, since studies have shown that they do not tend to consume fresh fruits and vegetables, and much of this population make use of cigarettes 
(which also require a greater use of AA) (Naidu 2003). The study by Subotkane et al. (1984) compared schizophrenic and non-schizophrenic patients consuming the same hospital diet. The results showed that the group suffering with SZ had significantly lower plasma levels of AA while fasting, and also showed an increased urinary excretion of AA after load testing with the vitamin. Even after four weeks of administrating AA (70 mg/ $\mathrm{kg}$ ), urinary excretion remained altered in patients with the disorder. However, Sivrioglu et al. (2007) showed that supplementation with AA (1000 mg), vitamin E (400U1) and omega-3 (180 mg EPA $+120 \mathrm{mg}$ DHA) in a group of 17 schizophrenic patients who were using haloperidol over a four month test period was beneficial towards both the positive and negative symptoms of SZ, as well as providing a sweetening of the side effects induced by haloperidol (Sivrioglu et al. 2007).

Creating improved resilience and neuronal plasticity will subsequently lead to cognitive and functional improvements which are extremely important in the treatment of SZ, resulting in a better quality of life for these patients. AA can be considered a new therapeutic approach for the treatment of this disorder, by helping to promote protection of the brain against oxidative damage and cellular alterations. Thus, the aim of this study was to evaluate the effects of AA supplementation on locomotor activity and the activity of the enzyme AChE in adult Wistar rats which had been subjected to an animal model of SZ induced by Ket.

\section{MATERIALS AND METHODS}

\section{ANIMALS}

Adult male Wistar rats with average weight between $250 \mathrm{~g}$ to $300 \mathrm{~g}$ were used in this study. The animals were obtained from the vivarium of Universidade do Extremo Sul Catarinense, and kept in cages with a 12 hour light/dark cycle. Food and water were available ad libitum, and the temperature was maintained at $22 \pm 1{ }^{\circ} \mathrm{C}$. The animals' health and diet acceptance were evaluated on a daily basis. The experiments were performed in the Neuroscience Laboratory at the Universidade do Extremo Sul Catarinense. All experimental procedures were performed in accordance with the International Recommendations for the Care and Use of Laboratory Animals, and also considering the recommendations for the use of animals outlined by the Brazilian Society of Neuroscience and Behavior (SBNeC). This project was implemented after approval by the Ethics Committee on Animal Use (CEUA) of the Universidade do Extremo Sul Catarinense (Protocol: 025/2013-2).

\section{EXPERIMENTAL DESIGN}

A total of 96 animals aged at 60 days were used in this study. The animals were separated into groups of 12 rats, and then divided into 8 experimental groups: Water+saline (Sal); Water+ketamine (Ket); AA 0.1 mg/kg+sal; AA 0.1mg/kg+ket; AA 1mg/kg+sal; AA 1mg/kg+ket; AA 10mg/kg+sal; AA $10 \mathrm{mg} / \mathbf{k g}+\mathbf{k e t}$. The doses of AA used in this study were based on the work done by Binfaré et al. (2009). AA was dissolved in distilled water, and this solution, which was freshly prepared before each administration, was given orally (p.o) by gavage in three different doses, daily for 14 days. Ketamine was prepared in saline at a volume of $1 \mathrm{~mL} / 100 \mathrm{~g}$ (Becker and Grecksch 2004, Imre et al. 2006, Tomiya et al. 2006). Injections of Ket or Sal were administered by (i.p) between the $9^{\text {th }}$ and $15^{\text {th }}$ days of the experiment. After a period of 30 minutes from the last injection of Ket or Sal, the animals were subjected to the behavioral test (locomotor activity), and then killed by decapitation. The animal's brain structures (the prefrontal cortex, hippocampus and striatum) were then carefully dissected for biochemical analysis of AChE activity.

\section{Behavioral Test}


Locomotor activity was scored using an Activity Monitor (Insight Equipment Laboratory) to measure the behavioral changes in the animals which were induced by ketamine, and may have been prevented by AA. The animals were assessed 30 min after the last injection of ketamine. This activity was conducted in a 40x60 $\mathrm{cm}$ enclosure made of acrylic, which is surrounded by walls approximately $50 \mathrm{~cm}$ in height. The monitor is surrounded by six parallel bars, each bar containing 16 infrared sensors that are able to detect the exact position and movements of the subject, so evaluating the detailed behavior of the animal under test. The animals were assessed for a period of 15 minutes (De Oliveira et al. 2009). The information detected by the sensors was then transmitted to a computer via the Open Source version of the Interbase 6:01 software (Activity Monitor - Insight Equipment Laboratory, Ribeirão Preto - São Paulo) for statistical analysis.

\section{BIOCHEMICAL ANALYSIS}

At the conclusion of the treatments, the animals were decapitated using a guillotine, and their brain structures (the prefrontal cortex, hippocampus and striatum) were carefully dissected for biochemical analysis of AChE enzymatic activity.

\section{PROTEIN DETERMINATION}

Protein was determined using the method of Lowry and colleagues (Lowry et al. 1951) where bovine serum albumin was used as a standard. All samples were analyzed in duplicate.

\section{AChE ACTIVITY}

The activity of AChE is based on the production of thiocholine by the hydrolysis of ACh. This is accompanied by a continuous reaction as thiol ion 5: 5-ditibis-2-nitrobenzoate (DTNB) produces the yellow colored anion 5-tol-2-nitro benzoic acid. The dissected brain tissue (the prefrontal cortex, striatum and hippocampus) was homogenized in phosphate buffer, 0.1 M pH. 8.0. Ach was added to the homogenate and the activity of DTNB was measured by taking absorbance readings every 30 seconds over a period of two minutes at $25^{\circ} \mathrm{C}$. The colorimetric reaction was observed in a spectrophotometer at $412 \mathrm{~nm}$ (Ellman et al. 1961).

\section{STATISTICAL ANALYSIS}

The programs used to perform the statistical analysis were the Statistics 7.0 and Graphpade Software packages, the latter of which was used to produce the graphics used in this report. Statistical analysis of the data was assessed using the Twoway analysis of variance (ANOVA) test, followed by the post hoc Newman Kewls test when F was significant. The results were shown as the mean \pm SEM. Statistical significance was set at $\mathrm{p}<0.05$.

\section{RESULTS}

\section{BEHAVIORAL TEST}

Figure 1 demonstrates the effect that the administration of AA had on locomotor activity in an animal model of SZ induced by Ket. Two-way ANOVA revealed a significant interaction between the variables: AA, Sal and Ket $[\mathrm{F}(3.68)=8.263$; $\mathrm{p}<0.01]$. There was a significant increase in the amount of locomotor activity in the animals that received Ket in comparison to the control group (Water+Sal) $(\mathrm{p}<0.05]$, indicating that Ket induces positive symptoms in an animal model of SZ. Supplementation of AA $(0.1,1$ and $10 \mathrm{mg} / \mathrm{kg})$ was able to prevent the hyperlocomotion induced by Ket $(\mathrm{p}<0.05)$. Additionally, AA alone did not manage to alter the behavioral results, indicating that the effects of AA seen in the animals treated with Ket were not associated with sedation.

\section{BIOCHEMICAL ANALYSIS}

The activity of the AChE enzyme within the prefrontal cortex, hippocampus and striatum are described in Figure 2 (panel A, B and C, 


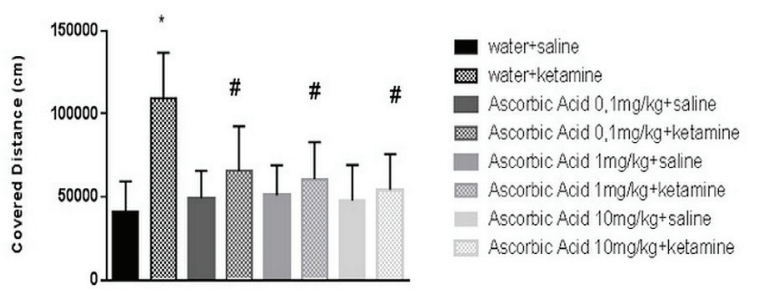

Figure 1 - Locomotor activity. The effects of supplementation with AA $(0.1,1$ and $10 \mathrm{mg} / \mathrm{kg}$, p.o) on locomotor activity in an animal model of SZ induced by Ket $(25 \mathrm{mg} / \mathrm{kg}$, i.p). Data are expressed as mean $\pm \operatorname{SEM}(\mathrm{N}=10-12) ;{ }^{*} \mathrm{p}<0.05$ difference from Water + Sal (control group); \# $\mathrm{p}<0.05$ difference from Water+Ket group.

respectively). Within the prefrontal cortex (Fig. 2a), hippocampus (Fig. 2b) and striatum (Fig. 2c), the two-way ANOVA test revealed a significant interaction between the variables: AA, Sal and Ket: $[\mathrm{F}(3.22)=7.85, \mathrm{p}<0.01],[\mathrm{F}(3.29)=5.72$, $\mathrm{p}<0.01]$ and $[\mathrm{F}(3.28)=5.23, \mathrm{p}<0.01]$, respectively. Our findings show that Ket increased the activity of the AChE enzyme in the prefrontal cortex and striatum $(p<0.01)$ when compared to the control group. Supplementation of AA $(0.1,1$ and $10 \mathrm{mg}$ / $\mathrm{kg}$ ) in association with Ket resulted in a significant decrease in the activity of $\mathrm{AChE}(\mathrm{p}<0.01)$ in relation to the ket group in all of the brain structures analyzed in this study, preventing an increase in the amount of damage caused by Ket. According to Fig. 2b, no effect was observed for Ket ( $\mathrm{p}=0.45)$ or any of dose of AA $(0.1 \mathrm{mg} / \mathrm{kg}, \mathrm{p}=0.51)$ and $(1 \mathrm{mg} / \mathrm{kg}, \mathrm{p}=0.63)$ on the levels of AChE activity in the hippocampus when compared to the control group, except for the results concerning AA supplementation $(10 \mathrm{mg} / \mathrm{kg}$, $\mathrm{p}<0.01$ ), where AA was able to increase the activity of this enzyme.

\section{DISCUSSION}

It is known that there is a direct correlation between deficiencies in AA and SZ. The hypothesis that this vitamin may be involved in the etiology of the disorder is based upon clinical studies which suggest that AA can improve the symptoms of SZ and help to reduce the levels of oxidative damage,
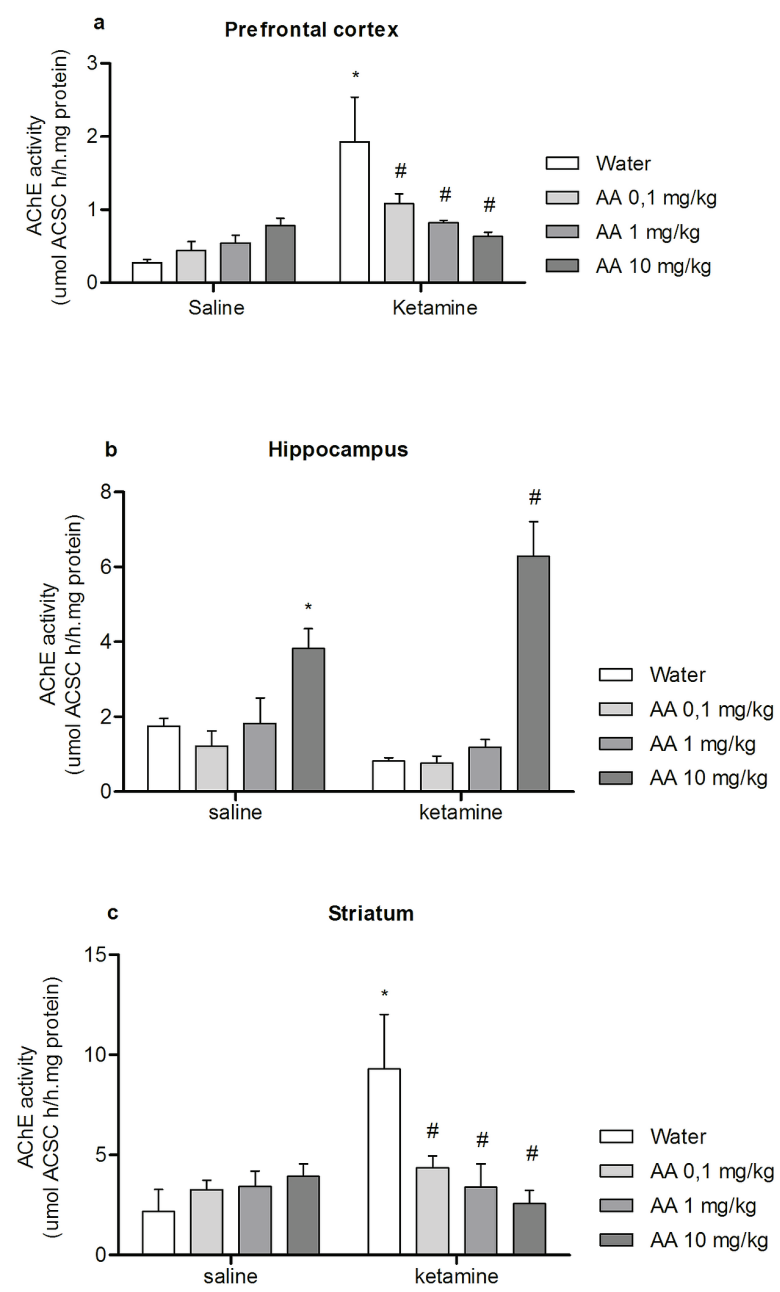

Figure 2 - Activity of the enzyme AchE. The effect of supplementation with AA $(0.1,1$ to $10 \mathrm{mg} / \mathrm{kg}, \mathrm{p} . \mathrm{o})$ on the activity of the enzyme AchE within different brain structures: panel a (prefrontal cortex); panel b (hippocampus) and panel c (striatum) in an animal model of SZ induced by Ket $(25 \mathrm{mg} /$ $\mathrm{kg}$, i.p). Data are expressed as mean $\pm \operatorname{SEM}(\mathrm{N}=5-6) ; * \mathrm{p}<0.01$ difference from the Water+Sal (control group); \# p $<0.01$ difference from the Water+Ket group.

mainly due to its powerful antioxidant action (Milner 1963). Emphasizing the hypothesis that schizophrenic patients require higher levels of AA when compared to healthy people, and knowing that the diets of individuals with mental illnesses are often deficient in vitamins, this could further aggravate the brain functions of these individuals (Popper 2001). Moreover, Dadheech et al. (2006) 
depict that schizophrenic patients have a reduction in their levels of AA, D-tocopherol and glutamine when compared to normal individuals.

In view of the serious adverse effects, poor patient adherence to antipsychotics and the low efficacy of these drugs (Gilmer et al. 2004, Schultz et al. 2007), it is essential to develop new strategies to prevent and / or provide adjunctive therapies that can reduce the adverse effects of treatment which are common with antipsychotics (Arvindakshan et al. 2003), such as weight gain, hypercholesterolemia and diabetes, amongst others (Ganguli 1999).

It has been shown that the administration of Ket in animals is capable of causing deficits similar to those observed in humans, which are associated with the loss of the animal's perception and an increase in locomotor activity (Gamma et al. 2012). In the present study, we tested the hypothesis that AA supplementation could prevent some schizophrenia-like phenotypes in an animal model of SZ, and that the antipsychotic effect elicited by AA is associated, at least in part, to decreases in the levels of redox dysregulation caused by NMDAR hypofunction.

The positive symptoms of SZ are characterized by hallucinations and delusions. In our animals, we observed an increase in the levels of locomotor activity induced by Ket, which was considered an altered behavior related to positive symptoms in an animal model of SZ (Gamma et al. 2012, Zugno et al. 2013). Krystal et al. (1994) also observed similar behavior in humans undergoing subanesthetic doses of Ket. This is an important parameter in evaluating the potential of any new therapeutic strategies that can alleviate the positive symptoms of SZ in an animal model (Chatterjee et al. 2012). Chronic NMDAR antagonism led to a marked increase of intracellular glutamate in the hippocampus. In addition, current proponents of the glutamatergic hypothesis postulate that hypofunctional NMDAR located on gamma-aminobutyric acid (GABA)ergic inhibitory interneurons disinhibit pyramidal neurons, leading to a paradoxical increase of glutamatergic activity within the prefrontal cortex and striatum, promoting the development of positive and negative symptoms (Stone et al. 2007, Nakazawa et al. 2012). An important neuromodulatory role of AA appears to involve the presynaptic re-uptake of glutamate. Thus, AA is capable of preventing the excitotoxic damage caused by an excess of extracellular glutamate, which would otherwise lead to the hyperpolarization of NMDAR, and subsequent neuronal damage. In this study, we found that AA $(0.1,1$ and $10 \mathrm{mg} /$ $\mathrm{kg}$ ) improved the hyperlocomotion induced by Ket, preventing the positive symptoms seen in an animal model of SZ. However, these complex interactions certainly require further investigations, especially if we consider that the amelioration of positive symptoms by antipsychotic drugs may be related to actions affecting different receptor systems or intracellular pathways.

$\mathrm{ACh}$ is a major neurotransmitter involved in cognitive function which is primarily mediated by nicotinic and muscarinic receptors. Thus, the cognitive deficits observed in schizophrenic patients can be related to the cholinergic system (Hasselmo 2006, Money et al. 2010). The results in this study show that the chronic administration of Ket $(25 \mathrm{mg} /$ $\mathrm{kg}$ ) increased the activity of $\mathrm{AChE}$ in the prefrontal cortex and striatum, while supplementation with AA $(0.1,1$ and $10 \mathrm{mg} / \mathrm{kg})$ prevented this increase in these same brain structures, showing AA was providing a protective effect. Taken together, our results indicate that the administration of AA prevented the positive symptoms of SZ, and was also able to reduce the activity of AChE observed in the animal model, thereby allowing the possible increase in levels of ACh seen in the synaptic cleft. These results corroborate the findings of Zugno et al. (2013). This data shows that Ket induced dysfunction of the cholinergic system in the same ways that have been observed in schizophrenic patients. AA can interfere with synaptic plasticity 
by improving neuronal signaling via its actions within important cellular processes and also due to its antioxidant function, thus acting against the cellular damage caused by Reactive Oxigen species (ROS) (Zugno et al. 2013).

Arvindakshan et al. (2003) demonstrated that there was a significant reduction in the clinical signs of SZ and an increase in the quality of life of schizophrenic patients that were supplemented with AA combined with D-tocopherol and omega 3 twice a day for a period of 4 months. Another study conducted by Dakhale et al. (2005) evaluated the effects of AA supplementation in schizophrenic patients treated with atypical antipsychotics for a period of eight weeks, and found that there was a reduction in the levels of oxidative damage, as well as an improvement in the scores on the Brief Psychiatric Rating Scale of SZ. A recent study has also shown that supplementation with AA ( $5 \mathrm{mg} / \mathrm{l})$ decreased the effects of neurotoxicity induced by aluminum by reducing the damage within neurotransmitters (serotonin, norepinephrine, dopamine), so decreasing the levels of apoptosis and restoring the morphology of the brain tissue in Nile catfish (Khalili and Hussein 2015). Moreover, Sepehri and Ganji (2016) showed that orogastric administrations of AA $(100 \mathrm{mg} / \mathrm{kg})$ were able to partially reduce the damage seen in the hippocampal CA1 region of pregnant rats exposed to lead. Furthermore, experimental studies have demonstrated that an increase in antioxidant capacity amplifies the excitatory postsynaptic potential, which improves synaptic plasticity (Sepehri et al. 2016) and cognitive ability (Liu et al. 2014). Data from this study demonstrates that an animal model of SZ induced by Ket was also able to induce changes in the cholinergic system, which were prevented by supplementation with AA. However, at its higher dose $(10 \mathrm{mg} / \mathrm{kg})$, AA alone or in combination with Ket showed an intriguing result due to the increases seen in the activity of the enzyme AChE within the hippocampus. This result shows the importance of pre-clinical studies in order to find a safe dose for the supplementation of AA that is capable of exhibiting its beneficial effects within psychiatric disorders. The importance of this work must also be emphasized, as no other studies have undertaken research into the preventive use of AA in animal models of SZ.

Analysis of the data from this study indicates that Ket alone is able to induce schizophreniclike behavior and increase the levels of $\mathrm{AChE}$ activity in the prefrontal cortex and striatum, while supplementation with AA showed a protective effect in preventing the behavioral and biochemical alterations induced by Ket in an animal model of SZ. These results suggest that this vitamin may be an adjuvant approach for the development of new therapeutic strategies within this psychiatric disorder. We suggest that this action may be due to its neuromodulatory properties, specifically, via the interaction of NMDAR hypofunction induced by Ket. However, future studies are necessary to confirm the intracellular and intercellular signaling processes of AA, which promote this important neuromodulatory action in an animal model of SZ. Moreover, new pre-clinical trials are needed to confirm the neuromodulatory action of AA in an animal model of SZ, and to give impetus to new clinical studies.

\section{ACKNOWLEDGMENTS}

The authors wish to thank the Universidade do Extremo Sul Catarinense for the opportunity to undertake this study, and allowing the use of the facilities within the university. They would also like to thank the entire team from the Neuroscience Laboratory for their hard work during the design, development, data analysis and preparation of this article. Finally, they offer thanks to the Conselho Nacional de Desenvolvimento Científico e Tecnológico $(\mathrm{CNPq})$ and the Fundação de Amparo à Pesquisa e Inovação do Estado de Santa Catarina 
(FAPESC), which are both agencies responsible for grants supporting this work.

\section{REFERENCES}

ARVINDAKSHAN MG, PRABHAKAR K, RANJEKAR DR AND EVANS SP. 2003. Supplementation with a combination of n-3 fatty acids and antioxidants (vitamins $\mathrm{E}$ and C) improves the outcome of schizophrenia. Schizophr Res 62: 195-204.

AYHAN Y ET AL. 2010. Differential effects of prenatal and postnatal expressions of mutant human DISC1 on neurobehavioral phenotypes in transgenic mice: evidence for neurodevelopmental origin of major psychiatric disorders. Mol Psychiatry 16: 293-306.

BAGUELIN-PINAUD A, ROBERT S, MÉNARD JF AND THIBAUT F. 2010. Prenatal exposure to tobacco and risk for schizophrenia: a retrospective epidemiological study. Compr Psychiatry 51: 106-109.

BECKER A AND GRECKSCH G. 2004. Ketamineinduced changes in rat behaviour: a possible animal model of schizophrenia. Test of predictive validity Prog Neuropsychopharmacol Biol Psychiatry 28(8): 1267-1277.

BENCHERIF M, STACHOWIAK MK, KUCINSKI AJ AND LIPPIELLO PM. 2012. Alpha7 nicotinic cholinergic neuromodulation may reconcile multiple neurotransmitter hypotheses of schizophrenia. Med Hypotheses 78: 594600.

BINFARÉ RW, ROSA AO, LOBATO KR, SANTOS AR AND RODRIGUESAL. 2009. Ascorbic acid administration produces an antidepressant-like effect: evidence for the involvement of monoaminergic neurotransmission. Prog Neuropsychopharmacol Biol Psychiatry 33(3): 530-540.

BOISON D, SINGER P, SHEN HY, FELDON J AND YEE BK. 2012. Adenosine hypothesis of schizophrenia Opportunities for pharmacotherapy. Neuropharmacology 62: 1527-1543.

BOWIE CR AND HARVEY PD. 2006. Schizophrenia from a neuropsychiatric perspective. Mt Sinai J Med 73: 993998.

CANEVER L, OLIVEIRA L, D'ALTOÉ DE LUCA R, CORREA PT, DE B FRAGA D, MATOS MP, SCAINI G, QUEVEDO J, STRECK EL AND ZUGNO AI. 2010. A rodent model of schizophrenia reveals increase in creatine kinase activity with associated behavior changes. Oxid Med Cell Longev 3: 421-427.

CHATTERJEE M, VERMA R, GANGULY S AND PALIT G. 2012. Neurochemical and molecular characterization of ketamine-induced experimental psychosis model in mice. Neuropharmacology 63: 1161-1171.

DADHEECH G, MISHRA S, GAUTAM S AND SHARMA P. 2006. Oxidative stress, -tocopherol, ascorbic acid and reduced glutathione status in schizophrenics. Indian $\mathrm{J}$ Clin Biochem 21: 218-222.

DAKHALE GN, KHANZODE SD, KHANZODE SS AND SAOJIA. 2005. Supplementation of vitamin $C$ with atypical antipsychotics reduces oxidative stress and improves the outcome of schizophrenia. Psychopharmacology 182: 494498.

DE OLIVEIRA L, FRAGA DB, DE LUCA RD, CANEVER L, GHEDIM FV, MATOS MP, STRECK EL, QUEVEDO J AND ZUGNO AI. 2011. Behavioral changes and mitochondrial dysfunction in a rat model of schizophrenia induced by ketamine. Metab Brain Dis 26: 69-77.

DE OLIVEIRA L, SPIAZZI CM, BORTOLIN T, CANEVER L, PETRONILHO F, MINA FG, DAL-PIZZOL F, QUEVEDO J AND ZUGNO AI. 2009. Different subanesthetic doses of ketamine increase oxidative stress in the brain of rats. Prog Neuro-Psychopharmacol Biol Psychiatry 33: 1003-1008.

ELLMAN GL, COURTNEY KD, ANDRES VJR AND FEATHER-STONE RM. 1961. A new and rapid colorimetric determination of acetylcholinesterase activity. Biochemical Pharmacology 7: 88-95.

FREEDMAN R. 2003. Schizophrenia. N Engl J Med 349: 1738-1749.

GAMA CS ET AL. 2012. Effects of omega-3 dietary supplement in prevention of positive, negative and cognitive symptoms: a study in adolescent rats with ketamine-induced model of schizophrenia. Schizophr Res 141: 162-167.

GANGULI R. 1999. Weight gain associated with antipsychotic drugs. J Clin Psychiatry 60: 20-24.

GILMER TP, DOLDER CR, LACRO JP, FOLSOM DP, LINDAMER L, GARCIA P AND JESTE DV. 2004. Adherence to treatment with antipsychotic medication and health care costs among Medicaid beneficiaries with schizophrenia. Am J Psychiatry 161: 692-699.

HASSELMO ME. 2006. The role of acetylcholine in learning and memory. Curr Opin Neurobiol 16: 710-715.

IMRE G, FOKKEMA DS, DEN BOER JA AND TER HORST GJ. 2006. Dose-response characteristics of ketamine effect on locomotion, cognitive function and central neuronal activity. Brain Res Bull 69(3): 338-345.

JAVITT DC. 2010. Glutamatergic theories of schizophrenia. Isr J Psychiatry Relat Sci 47: 4-16.

KHALILI SR AND HUSSEIN MM. 2015. Neurotransmitters and neuronal apoptotic cell death of chronically aluminum intoxicated Nile catfish (Clarias gariepinus) in response to ascorbic acid supplementation. Neurotoxicology 51: 184191.

KRYSTAL JH, KARPER LP, SEIBYL JP, FREEMAN GK, DELANEY R, BREMNER JD, HENINGER GR, BOWERS JR MB AND CHARNEY DS. 1994. Subanesthetic effects of the noncompetitive NMDA 
antagonist, ketamine, in humans. Psychotomimetic, perceptual, cognitive, and neuroendocrine responses. Arch Gen Psychiatry 51: 199-214.

LIPSKA BB AND WEINBERGER D. 2000. To Model a Psychiatric Disorder in Animals: Schizophrenia As a Reality Test. Neuropsychopharmacology, p. 223-239.

LIU X, ZHANG Y, LI J, WANG D, WU Y, LI Y, LU Z, YU SC AND YANG X. 2014. Cognitive deficits and decreased locomotor activity induced by single-walled carbon nanotubes and neuroprotective effects of ascorbic acid. Int J Nanomedicine 11: 823-839.

LOWRY OH, ROSEBROUGH NJ, FARR AL AND RANDALL RJ. 1951. Protein measurement with the Folin phenol reagent. J Biol Chem 193: 265-275.

MARI JJ AND LEITÃO RJ. 2000. A epidemiologia da esquizofrenia. Rev Bras Psiquiatr 22: 15-17.

MARSMAN A, VAN DEN HEUVEL MP, KLOMP DW, KAHN RS, LUIJTEN PR AND HULSHOFF POL HE. 2013. Glutamate in schizophrenia: a focused review and meta-analysis of ${ }^{1} \mathrm{H}-\mathrm{MRS}$ studies. Schizophr Bull 39: 120-129.

MECHERI A, SAOUD M, KHIARI G, D'AMATO T, DALERY J AND GAHA J. 2001. Glutaminergic hypothesis of schizophrenia: clinical research studies with ketamine. Encephale 27: 53-59.

MELTZER HY AND MCGURK SR. 1999. The effects of clozapine, risperidone, and olanzapine on cognitive function in schizophrenia. Schizophr Bull 25: 233-255.

MEYER U AND FELDON J. 2010. Epidemiology-driven neurodevelopmental animal models of schizophrenia. Progress in Neurobiology 90: 285-326.

MILNER G. 1963. Ascorbic acid in chronic psychiatric patients: A controlled trial. Br J Psychiatry 109: 294-299.

MONEY TT, SCARR E, UDAWELA M, GIBBONS AS, JEON WJ, SEO MS AND DEAN B. 2010. Treating schizophrenia: novel targets for the cholinergic system. CNS Neurol Disord Drug Targets 9: 241-256.

NAGAI T, IBI D AND YAMADA K. 2011. Translational Research in Neurodevelopmental Disorders: Development of Etiology-Based Animal Models: Animal Model for Schizophrenia That Reflects Gene-Environment Interactions. Biol Pharm Bull 34: 1364-1368.

NAIDU KA. 2003. Vitamin C in human health and disease is still a mystery? An Overview Nutr J 2: 7.

NAKAZAWA K ET AL. 2012. GABAergic interneuron origin of schizophrenia pathophysiology. Neuropharmacol 62: 1574-1583.

POPPER CW. 2001. Do vitamins or minerals (apart from lithium) have mood-stabilizing effects? J Clin Psychiatry 62: 933-935.

REDDY R, KESHAVAN M AND YAO JK. 2003. Reduced plasma antioxidants in first-episode patients with schizophrenia. Schizophr Res 62: 205-212.
RUMELIN A, HUMBERT T, LUHKER O, DRESCHER A AND FAUTH U. 2005. Metabolic clearance of the antioxidant ascorbic acid in surgical patients. J Surg Res 129: 46-51.

SCHORAH CJ, MORGAN DB AND HULLIN RP. 1983. Plasma vitamin $\mathrm{C}$ concentrations in patients in a psychiatric hospital. Human Nutr Clin Nutr 37: 447-452.

SCHULTZ SH, NORTH SW AND SHIELDS CG. 2007. Schizophrenia: a review. Am Fam Physician 75: 18211829.

SEPEHRI H AND GANJI F. 2016. The protective role of ascorbic acid on hippocampal CA1 pyramidal neurons in a rat model of maternal lead exposure. J Chem Neuroanat 16(74): 5-10.

SIVRIOGLU EY, KIRLI S, SIPAHIOGLU D, GURSOY B AND SARANDÖL E. 2007. The impact of omega-3 fatty acids, vitamins $\mathrm{E}$ and $\mathrm{C}$ supplementation on treatment outcome and side effects in schizophrenia patients treated with haloperidol: an open-label pilot study. Prog Neuropsychopharmacol Biol Psychiatry 31: 1493-1499.

SMYTHIES JR. 1996. The role of ascorbate in brain: Therapeutic implications. J Royal Soc Med 89(5): 241.

SMYTHIES JR. 1999. The role of ascorbate in brain: Therapeutic implications. J Royal Soc Med 89: 241.

SMYTHIES JR. 2000. Redox aspects of signaling by catecholamines and their metabolites. Antioxid Redox Signal 2: 575-583.

SOREQ H AND SEIDMAN S. 2001. Acetylcholinesterase-new roles for an old actor. Nat Rev Neurosci 2: 294-302.

STONE JM, MORRISON PD AND PILOWSKY LS. 2007. Glutamate and dopamine dysregulation in schizophrenia-a synthesis and selective review. J Psychopharmacol 21: 440-452.

SUBOTICANEC K, KULCAR Z, TURCIN R, SILA A, FOLNEGOVIĆ-SMALC V, MEŞTROVIĆ B AND BUZINA R. 1984. Vitamin C and schizophrenia. Acta Med lugosl 38: 299-308.

TOMIYA M, FUKUSHIMA T, KAWAI J, AOYAMA C, MITSUHASHI S, SANTA T, IMAI K AND TOYO'OKA T. 2006. Alterations of plasma and cerebrospinal fluid glutamate levels in rats treated with the N-methylD-aspartate receptor antagonist, ketamine. Biomed Chromatogr 20(6-7): 628-633.

VOSS B, THIENEL R, LEUCHT S AND KIRCHER T. 2008. Therapy of cognitive deficits in schizophrenia with acetylcholinesterase inhibitors. A systematic overview. Nervenarzt 79: 47-48,50-52,54-59.

ZUGNO AI ET AL. 2013. Rivastigmine reverses cognitive deficit and acetylcholinesterase activity induced by ketamine in an animal model of schizophrenia. Metab Brain Dis 28: 501-508. 